\title{
A Dynamic One-Equation Subgrid Model for Simulation of Flow around a Square Cylinder
}

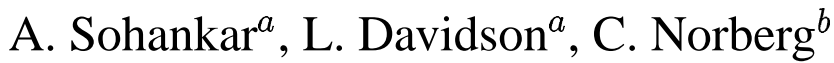 \\ ${ }^{a}$ Thermo and Fluid Dynamics, Chalmers University of Technology, \\ S-412 96, Göteborg, Sweden \\ ${ }^{b}$ Division of Heat Transfer, Lund Institute of Technology, Box 118, \\ S-221 00, Lund, Sweden
}

\begin{abstract}
Large Eddy Simulations (LES) of flow around a square cylinder at $R e=22 \times 10^{3}$ are performed employing a dynamic one-equation subgrid model. An implicit fractional step method finite-volume code with second-order accuracy in space and time is used. By using a periodic boundary condition the spanwise dimension is four times the side length of the cylinder. Some global quantities, such as the dominating wake frequency (Strouhal number) and the mean and RMS values of lift and drag, are computed. Also, a series of time- and spanwise-averaged resolved velocities and turbulent stresses are provided for comparison with experiments. The influence of a finer grid on the results is investigated. Present results are in general agreement with experiments and by using a finer spatial resolution an even better agreement is found. The dynamic one-equation subgrid model is found to be stable and no sign of the numerical problems usually encountered with the standard dynamic SGS-model is observed.
\end{abstract}

\section{Introduction}

Turbulence is present in a very wide variety of fbws of engineering problems involving transportation, energy conversion systems and wind engineering area, e.g fbw past bluff bodies. In other words, turbulence in nature is the rule and not the exception.

Thus, the issue of how to handle the influence of the turbulence, where diffusion of momentum and heat are much stronger than laminar fbws, is of great importance. Many turbulence models have been developed, ranging from the most simple, eddy viscosity models to high resolution Large Eddy Simulation (LES). When using LES in the context of the volume average approach (box fi lter), the time-dependent, three-dimensional Navier-Stokes equations are solved. In this method, the largest scales are resolved numerically, while the unresolved scales must be modeled with a subgrid scale model (SGS).

The subject of fbw past bluff bodies, such as circular and square cylinders, is of relevance to technical problems associated with energy conversion, structural design and acoustic emissions. In recent years, researchers attention has turned to the use of LES for studying turbulent 
fbw around bluff bodies. Also recently, this fbw case was studied experimentally by Lyn and Rodi [14] and Lyn et al. [14, 13].

A LES Workshop was held in June 1995 in Germany, and the results of this workshop were published in a recent paper by Rodi et al. [22]. The same fbw was considered as test case LES2 at the First ERCOFTAC Workshop on Direct and Large Eddy Simulation in March 1994. Seven groups took part in LES2 exercise and the results of this exercise were reported by Voke [30]. The reason for the focus on LES for the study of fbw around bluff bodies has to do with poor results when using statistical turbulence models. Most probably this has to do with complicating factors such as massive fbw separation, streamline curvature, transition from laminar to turbulent fbw, recirculation, vortex shedding and perhaps most important, the existence of inherent three-dimensional fbw structures [20, 21, 31].

\section{Configuration and Numerical Details}

The fbw is described in a Cartesian coordinate system $(x, y, z)$, in which the $x$-axis is aligned with inlet fbw direction, the $z$-axis is parallel with the cylinder axis and the $y$-axis is perpendicular to both directions, as shown in Fig. 1. A fi xed two-dimensional square cylinder with a side $d$ is exposed to a constant free stream velocity $U_{\infty}$. An incompressible fbw with constant fluid properties is assumed. The Reynolds number is defi ned as $R e=U_{\infty} d / \nu$. All geometrical lengths are scaled with $d$. Scaling with $d$ also applies to the Strouhal number, $S t=f_{S} d / U_{\infty}$, where $f_{S}$ is the shedding frequency, and for all forces. Velocities are also scaled with $U_{\infty}$, and physical times with $d / U_{\infty}$. In $y$-direction, the vertical distance between the upper and lower walls, $H$, defi nes the solid blockage of the confi ned fbw (blockage parameter, $\beta=1 / H$ ).

The main objective of the present study was the examination of a new dynamic one-equation subgrid-scale model, for Large Eddy Simulation of fbw around a square cylinder at $R e=$ $22 \times 10^{3}$. Also, the influence of fi ner grid on the results is investigated. A short review on LES mathematical formulation and dynamic one-equation subgrid scale model that is used in this work is presented in the next section.

An incompressible fi nite volume code [5], which is based on a fractional-step technique, is used employing a non-staggered grid arrangement. The scheme is implicit in time, and a second order Crank-Nicolson scheme is used. All terms are discretized using the second-order central differencing scheme. The time-marching calculations are started with the flid at rest, and a constant time step $\Delta t=1 / 40=0.025$ is used for all simulations.

Two calculations are carried out for the resolution of $185 \times 105 \times 25$ (Case I) and $265 \times 161 \times$ 25 (Case II) grid points in the $(x, y, z)$ direction. Outside a region from the body which extends two units upstream, downstream and sideways (in the $x$ - and $y$-directions), the grid distribution is uniform with a constant cell size, $\Delta$. Downstream of the body, $\Delta$ was set to 0.16 and 0.10 

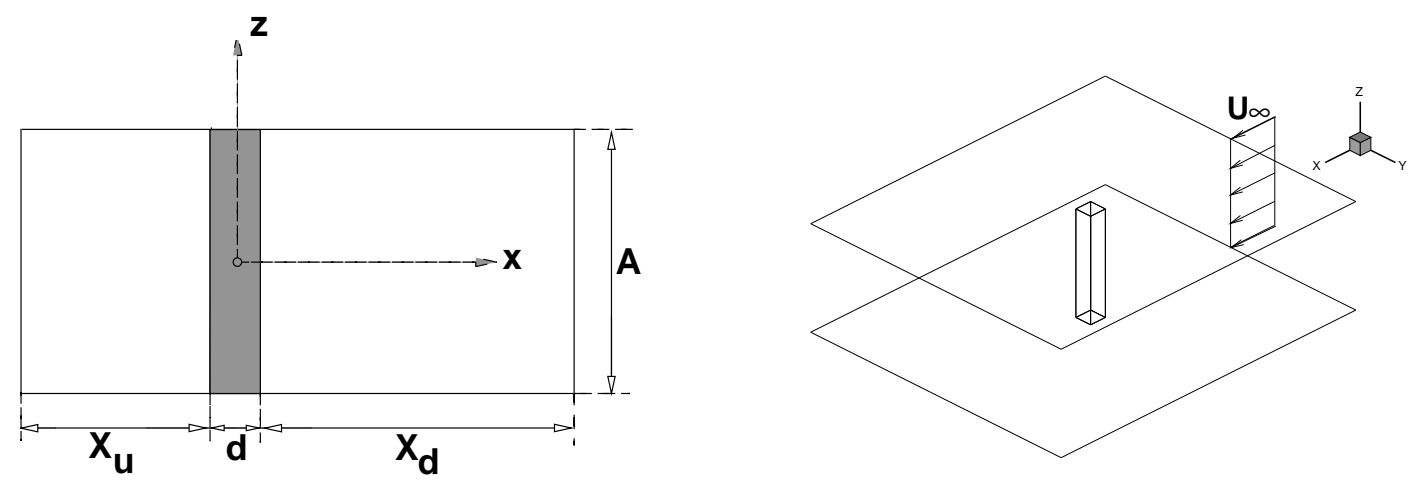

Figure 1: Flow confi guration.

for Cases I and II, respectively. In other parts, $\Delta=0.25$ for Case I and $\Delta=0.16$ for Case II. The distance from the cylinder surface to the nearest grid point defi nes $\delta$. For all calculations in this study, $\delta \approx 0.008$. The hyperbolic tangent function was used for stretching the cell sizes between these limits $\left(\delta\right.$ and $\Delta$ ). A uniform grid with a distance of $\Delta_{z}$ between nodes was used in the spanwise direction ( $z$-direction, (computational) spanwise dimension $A$ ). In this study, $\Delta_{z}=0.167$. The number of nodes distributed over one unit length of the cylinder surface was set equal to 25 and 33 for all sides of the body in Cases I and II, respectively.

The following boundary conditions were used. A uniform fbw was prescribed at the inlet, which is located $X_{u}$ units upstream of the cylinder. At the outlet, located $X_{d}$ units downstream of the cylinder, the convective boundary condition was used for all velocity components. No-slip conditions were prescribed at the body surfaces. Symmetry conditions simulating a frictionless wall were used at the upper and lower boundaries. A periodic boundary condition was used in the spanwise direction. The normal derivative for the pressure was set to zero at all boundaries. In the present study, $X_{u}, X_{d}, H$ and $A$ were set to 7.4, 15.8, 15.7 and 4, respectively, see Fig. 1.

\section{The Dynamic One-Equation Model}

The success of LES depends on how accurately the SGS stresses are modeled. The Smagorinsky model [23] is a traditional and relatively simple model. In this model, the proportionality factor in the defi nition of the subgrid eddy viscosity, $C$ or $C_{S}\left(C=C_{S}^{2}\right)$, is a constant value that must be specifi ed prior to a simulation. The weak point of this model has to do with the fact that it is not suitable to use a constant that is not really a single universal constant. In other words, the Smagorinsky coeffi cient is fbw-dependent. It is not a constant, and it varies in time and space. This model has some limitations and some attempts have thus been performed to improve it $[16,19]$. In addition, the Smagorinsky model is absolutely dissipative and cannot account 
for backscatter, energy is always transfered from the large to small scales. Dynamic models, which are capable of removing some of the drawbacks of the Smagorinsky model, are a suitable alternative. In these models, $C$ in the subgrid eddy viscosity is not an arbitrarily constant specifi ed a priori, but is calculated during the computation process. One of the drawbacks of the dynamic model is the numerical instability associated with the negative values and large variation of the $C$ coeffi cient.

Recently a new dynamic one-equation subgrid model was presented [3]. For convenience, the model is briefly described below. The modeled $k_{s g s}$ equation can be written

$$
\frac{\partial k_{s g s}}{\partial t}+\frac{\partial}{\partial x_{j}}\left(\bar{u}_{j} k_{s g s}\right)=\frac{\partial}{\partial x_{j}}\left(\left\langle C^{k}\right\rangle_{x y z} \Delta k_{s g s}^{\frac{1}{2}} \frac{\partial k_{s g s}}{\partial x_{j}}\right)+2 \nu_{s g s} \bar{S}_{i j} \bar{S}_{i j}-C_{*}^{k} \frac{k_{s g s}^{\frac{3}{2}}}{\Delta}
$$

In the production term, the dynamic coeffi cient $C^{k}$

$$
P_{k_{s g s}}=-\tau_{i j}^{a} \bar{u}_{i, j}, \quad \bar{S}_{i j}=\frac{1}{2}\left(\frac{\partial \bar{u}_{i}}{\partial x_{j}}+\frac{\partial \bar{u}_{j}}{\partial x_{i}}\right), \quad \tau_{i j}^{a}=-2 C^{k} \Delta k_{s g s}^{\frac{1}{2}} \bar{S}_{i j}=-2 \nu_{s g s} \bar{S}_{i j}
$$

is computed in a way similar to that used in the standard dynamic model $[7,8,10,9]$, i.e.

$$
\begin{aligned}
C^{k} & =-\frac{\mathcal{L}_{i j} M_{i j}}{2 M_{i j} M_{i j}}, \quad \mathcal{L}_{i j}={\overparen{\bar{u}_{i} \bar{u}_{j}}}-\widehat{\bar{u}}_{i} \widehat{\bar{u}}_{j} \\
K & =\widehat{k}_{s g s}+\frac{1}{2} \mathcal{L}_{i i}, \quad M_{i j}=\overparen{\Delta} K^{\frac{1}{2}} \widehat{S}_{i j}-\Delta \overbrace{k_{s g s}^{\frac{1}{2}} \bar{S}_{i j}}
\end{aligned}
$$

where $\mathcal{L}_{i j}$ denotes the dynamic Leonard stresses, and where $K \equiv \frac{1}{2} T_{i i}$ is the subgrid kinetic energy on the test level [10, 9, 4].

The subgrid turbulent kinetic energy, $k_{s g s}$, is essentially a local quantity. Indeed, the Smagorinsky model is based on the assumption of local equilibrium of $k_{s g s}$, i.e. $P_{k_{s g s}}-\epsilon_{k_{s g s}}=0$. A slightly better assumption to estimate $C_{*}^{k}$ in the dissipation term would be to assume that the fi ltered right-hand side of the $k_{s g s}$ equation is equal to that of the $K$ equation, i.e.

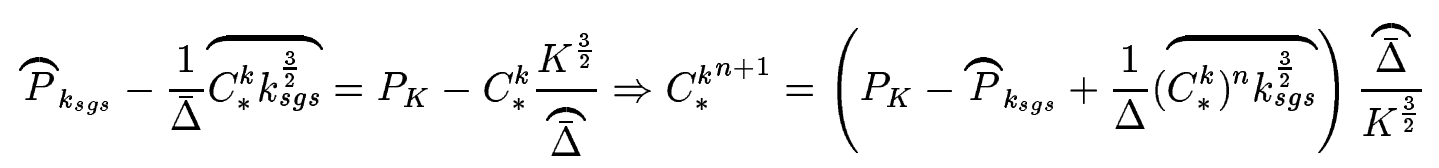

The dissipation cannot be negative, which requires that we limit $\left(C_{*}^{k}\right)^{n}$ to positive values, i.e. $C_{*}^{k} \geq 0$. To ensure numerical stability, a constant value of $C^{k}$ in space $\left(\left\langle C^{k}\right\rangle_{x y z}\right)$ is used in the momentum equations. This is determined by requiring that the production in the whole computational domain should remain the same, i.e.

$$
\left\langle 2 C^{k} \Delta k_{s g s}^{\frac{1}{2}} \bar{S}_{i j} \bar{S}_{i j}\right\rangle_{x y z}=2\left\langle C^{k}\right\rangle_{x y z}\left\langle\Delta k_{s g s}^{\frac{1}{2}} \bar{S}_{i j} \bar{S}_{i j}\right\rangle_{x y z}
$$


The idea is to include all local dynamic information through the source terms of the transport equation for $k_{s g s}$. This is probably physically more sound since large local variations in $C^{k}$ appear only in the source term, and the effect of the large fluctuations in the dynamic coeffi cients will be smoothed out in a natural way. In this way, it turns out that the need to restrict or limit the dynamic coeffi cient is eliminated altogether.

\section{Results and Discussion}

Calculations are carried out for two resolutions of $185 \times 105 \times 25$ (Case I) and $265 \times 161 \times 25$ (Case II) grid points in the $(x, y, z)$ direction on one SGI ORIGIN 2000 machine. The transient period before the fully developed state is achieved, is about 50 time units, which is greater than 6 shedding periods. The CPU time on one processor per time step and grid point is about $2.2 \times 10^{-4}$ CPU seconds for Case I. The number of iterations per time step is about 2 . The time-averaged quantities are calculated during about 20 shedding cycles.

In the present study, the global results (time- and spanwise-averaged values) of these two cases are compared with the experimental ones [12, 29, 2, 15, 14, 13, 17] and numerical results presented at the LES Workshop [22] and LES2 exercise [30]. A summary of the global results is provided in Table 1. Also, a series of time- and spanwise-averaged resolved velocity and turbulent stresses are provided for comparison with experiments.

Table 1: Summary of global results for different cases and comparison with experiments. Please note that different blockages ( $\beta=2-7 \%$ ), turbulence intensities ( $T u \leq 2 \%$ ), aspect ratios $(A R=10-51)$ and end conditions are used in the experiments.

\begin{tabular}{cccccccc}
\hline Results & $R e / 10^{3}$ & $S t$ & $C_{D}$ & $-C_{p b}$ & $C_{L^{\prime}}$ & $C_{D^{\prime}}$ & $l_{r}$ \\
\hline Case I & 22 & 0.129 & 2.25 & 1.55 & 1.50 & 0.20 & 1.0 \\
Case II & 22 & 0.131 & 2.32 & 1.63 & 1.49 & 0.21 & 1.1 \\
\hline \hline Ref. 22 & 22 & $0.066-0.14$ & $1.66-2.77$ & - & $0.38-1.79$ & $0.1-0.27$ & $0.89-2.96$ \\
Ref. 30 & 21.4 & $0.13-0.161$ & $2.03-2.78$ & - & $1.03-1.68$ & $0.12-0.36$ & $1.02-1.61$ \\
\hline \hline EXP. & & & & & & & \\
\hline \hline Ref. 17 & 13 & 0.132 & 2.16 & 1.43 & - & - & - \\
Refs. 13,14 & 21.4 & 0.13 & 2.1 & - & - & - & 1.38 \\
Ref. 2 & 22 & - & 2.0 & - & 0.5 & - & - \\
Ref. 15 & 23 & - & - & - & 1.3 & - & - \\
Ref. 29 & 100 & 0.118 & 2.05 & 1.32 & 1.32 & 0.17 & - \\
Ref. 12 & 176 & 0.122 & 2.04 & 1.3 & 1.23 & 0.23 & - \\
\hline
\end{tabular}


In the case of fbw around square cylinders, some of the global quantities for high Reynolds numbers are approximately independent of the variation in the Reynolds number. For example, Bearman \& Obasaju [1] reported that the pressure drag and lift forces are independent of Reynolds numbers above $R e=2 \times 10^{4}$. Within $4 \times 10^{3} \leq R e \leq 40 \times 10^{3}$ and based on the low-blockage $(\beta \leq 5 \%)$ and low-freestream turbulence $(T u \leq 0.5 \%)$ experiments by Okajima (1982) [18], Igarashi (1984) [11] and Norberg (1993) [17] the Strouhal number is $S t=0.129 \pm 0.005$. At around $R e=22 \times 10^{3}$ the above experiments have $S t=0.132 \pm 0.001$. It is also observed in Table 1 that the variations in the Strouhal number, mean drag and base pressure coeffi cients with Reynolds numbers are small in a large range of Reynolds numbers $\left(13 \times 10^{3} \leq R e \leq 176 \times 10^{3}\right)$.

Table 1 gives the Strouhal number, mean drag, base pressure, RMS lift and drag coeffi cients for different SGS models, together with the experiment and numerical results. The present Strouhal numbers were calculated from the fluctuating lift signal. The Strouhal number in the present study is in agreement with the experiments. As can be seen, the Strouhal number is not very sensitive to different resolutions. This fi nding is similar to that reported by Rodi et al. [22] and Sohankar [24], who also found that the Strouhal number is not very sensitive to the parameters of the simulation.

The experimental results for the mean drag coeffi cient are reported in the range of $C_{D}=$ $2.0-2.16$, for large range of Reynolds numbers, see Table 1 . The mean drag for present study is about $4 \%$ and $7 \%$ higher than the upper range of the experimental results for the Cases I and II, respectively. From Table 1, the base suction coeffi cients $\left(-C_{p b}\right)$ are: 1.55 and 1.63 for the Cases I and II , respectively. A higher base suction corresponds to a higher drag because the pressure on the upstream of body is similar for all cases and experiments, see e.g Ref. [24]. Rodi et al. [22] reported that the simulations that used no-slip boundary conditions for body produce a higher drag and lower recirculation length, $l_{r}$, than those that used a wall function boundary condition. In this study, a no-slip boundary condition is used.

As is seen in Table 1, the experimental results for RMS lift and drag display considerably variations. The RMS lift and drag coeffi cients in Table 1, in the present study, are spanwiseaveraged values. For RMS lift the ratio between the spanwise-averaged and the spanwise-mean sectional value was 0.99 for the two cases. For RMS drag the corresponding ratios were 0.76 and 0.73 for the Cases I and II, respectively. The experimental $C_{L^{\prime}}$ and $C_{D^{\prime}}$ in Table 1 are all sectional values. The experimental RMS lift and drag coeffi cients are reported in the range of $C_{L^{\prime}}=0.5-1.32$ and $C_{D^{\prime}}=0.17-0.23$. The results of the LES Workshop and LES2 exercise also show a large variation in $C_{L^{\prime}}$ and $C_{D^{\prime}}$, see Table 1. For example, the range of predicted variations in RMS lift and drag is reported to be $C_{L^{\prime}}=0.38-1.79$ and $C_{D^{\prime}}=0.1-0.27$. These large variations are dependent on a number of factors, such as different SGS models, grids, boundary conditions and so on. The present results for RMS lift are higher than experimental ones. The values for the RMS drag of this study are within the scatter of the reported 
experimental results, see Table 1. It is worth mentioning that, using fi ner grid (Case II) has little influence on RMS lift and drag. The blockage effect, different turbulence intensities, length scale of incoming turbulence, end conditions, aspect ratio and Reynolds number dependency could be reasons for the scatter of the reported experimental results, see e.g. [2, 15]. Previous works by the present authors [25, 26, 27, 28] have shown that the RMS lift and drag coeffi cients are more sensitive than mean values to various numerical and physical parameters. The experimental and numerical results in Table 1 confi rm this fi nding.

Figs. 2 and 3 compare the time- and spanwise-averaged resolved-velocity and Reynolds stresses along the centerline of the wake, and along $y$ direction at the position of two diameter downstream of the body, with experimental results $[14,13]$. The streamwise velocity $\bar{u}$ at the centerline of the wake is shown in Fig. 2. The minimum streamwise velocity and the extent of the recirculation region of present study are lower than the experimental values. The recovery of the streamwise velocity levels off at approximately 0.75 and 0.7 for Cases I and II, respectively, which is higher than the experiments, which is at about 0.6. Of these two cases, the finer resolution (Case II) has the larger recirculation length, $l_{r}$, larger negative streamwise velocity in the recirculation region and lower recovery of the streamwise velocity along centerline which show better agreement with experiment. This fi nding shows that a part of difference between experiment and numerical simulation for the recovery of the centerline velocity and $l_{r}$, which was also noted in Refs. [22, 30], is due to using a poor resolution.

The time- and spanwise-averaged of the different components of resolved Reynolds normal $\left\langle u^{\prime} u^{\prime}\right\rangle_{t z},\left\langle v^{\prime} v^{\prime}\right\rangle_{t z}$ and shear $\left.\left\langle u^{\prime} v^{\prime}\right\rangle_{t z}\right)$ stresses are shown in Fig. 2. Please note that the resolved time- and spanwise-averaged shear stress, $\left\langle u^{\prime} v^{\prime}\right\rangle_{t z}$, should be zero along the centerline due to symmetry. The predictions of normal stresses $\left(\left\langle u^{\prime} u^{\prime}\right\rangle_{t z}\right.$ and $\left.\left\langle v^{\prime} v^{\prime}\right\rangle_{t z}\right)$ along the centerline have relatively good agreement with experiment, especially for the fi ner resolution (Case II). The peak of the simulated normal stresses take places slightly closer to the body and with lower values than experiment for Case I, while better agreement with experiment is seen for Case II.

Fig. 3 shows profi les of velocity and stresses at position of two diameter downstream of the body. As is seen, there are good agreement with experiment for the shape and values of velocity and stresses especially for the fi ner resolution (Case II). In general, by referring to Figs. 2 and 3 and other results which are not shown in this paper (see Ref. [24]), a relatively good agreement with experiment for different components of velocity and stresses is found. This agreement is very good for the fi ner resolution (Case II).

As explained in the previous section, the local dynamic coeffi cient, $C$, for the standard dynamic model has a large variation in space and time. This large oscillation of $C$ enters directly in the momentum equations via the subgrid eddy viscosity, which can enhance numerical instability problems, see Ref. [24]. For example, in Ref. [24], the largest negative value for $C$ in the order of 7 was reported. In the one-equation model, on the other hand the local dynamic coeffi cient, $C^{k}$, enters in the source terms of the turbulent kinetic subgrid energy equation. To 
ensure numerical stability, a constant value of $C^{k}$ in space $\left(\left\langle C^{k}\right\rangle_{x y z}\right)$ is used in the momentum equations, which is determined by Eq. 5. This procedure increases the numerical stability by preventing a large oscillation in solution of the momentum equations. The time history of $C^{k}$ for two chosen points at midspan of the body $(z=0)$ on the centerline, one upstream of the body $(x=-0.64)$ and the second one downstream of the body $(x=6.35)$, are shown in Fig. 4(left). The range of variation of $C^{k}$ with time is much smaller upstream than downstream of the body. Interestingly, the $C^{k}$ has a negative value for all times at point $x=-0.64$ with the time-averaged value of -0.07 . The negative values of $C^{k}$ make the production term in the turbulent kinetic subgrid energy become negative, see Eq. 3. The negative production term means that backscattering occurs. The time history of $\left\langle C^{k}\right\rangle_{x y z}$ is shown in Fig. 4(right). The range of variation of $\left\langle C^{k}\right\rangle_{x y z}$ with time is between about 0.075 and 0.10 with a time-averaged value of 0.085 (dashed line). This time-averaged value is different for different fbw confi gurations. For example, the value for recirculating fbw is reported to be 0.04 [3]. For transitional fbw behind a backward-facing step, a value of 0.07 was found [6]. Due to the relatively small variation of the homogeneous time-dependent dynamic coeffi cient, no sign of numerical problems was observed.

\section{Conclusions}

Large Eddy Simulations (LES) of the fbw around a square cylinder at $R e=22 \times 10^{3}$ are performed employing a dynamic one-equation subgrid model. By comparing computed global quantities, mean fbw with experiments, this simulation shows that the one-equation subgrid scale model gives relatively good agreement with experiments. This agreement is very good for the fi ner resolution.

Due to the relatively small variation of the homogeneous time-dependent dynamic coeffi cient in this model, no sign of numerical problems, which is seen for standard dynamic model, was observed.

The local dynamic coeffi cient of this model $\left(C^{k}\right)$ becomes negative at some point close to the upstream of the body at all the time. The negative values of $C^{k}$ make the production term in the turbulent kinetic subgrid energy become negative. The negative production term means that backscattering occurs.

\section{References}

[1] P. W. Bearman and E. D. Obasaju. J. Fluid Mech., 119:297-321, 1982.

[2] C. M. Cheng and R. H. Chen. J. Wind Eng. Ind. Aero, 41:739-749, 1992.

[3] L. Davidson. In 11th Int. Symp. on Turbulent Shear Flow, Grenoble, 3, pages 26.1-26.6, 1997.

[4] L. Davidson. Rept. 97/11, Dept. of Thermo and Fluid Dynamics, Chalmers Univ. of Tech., 1997. 

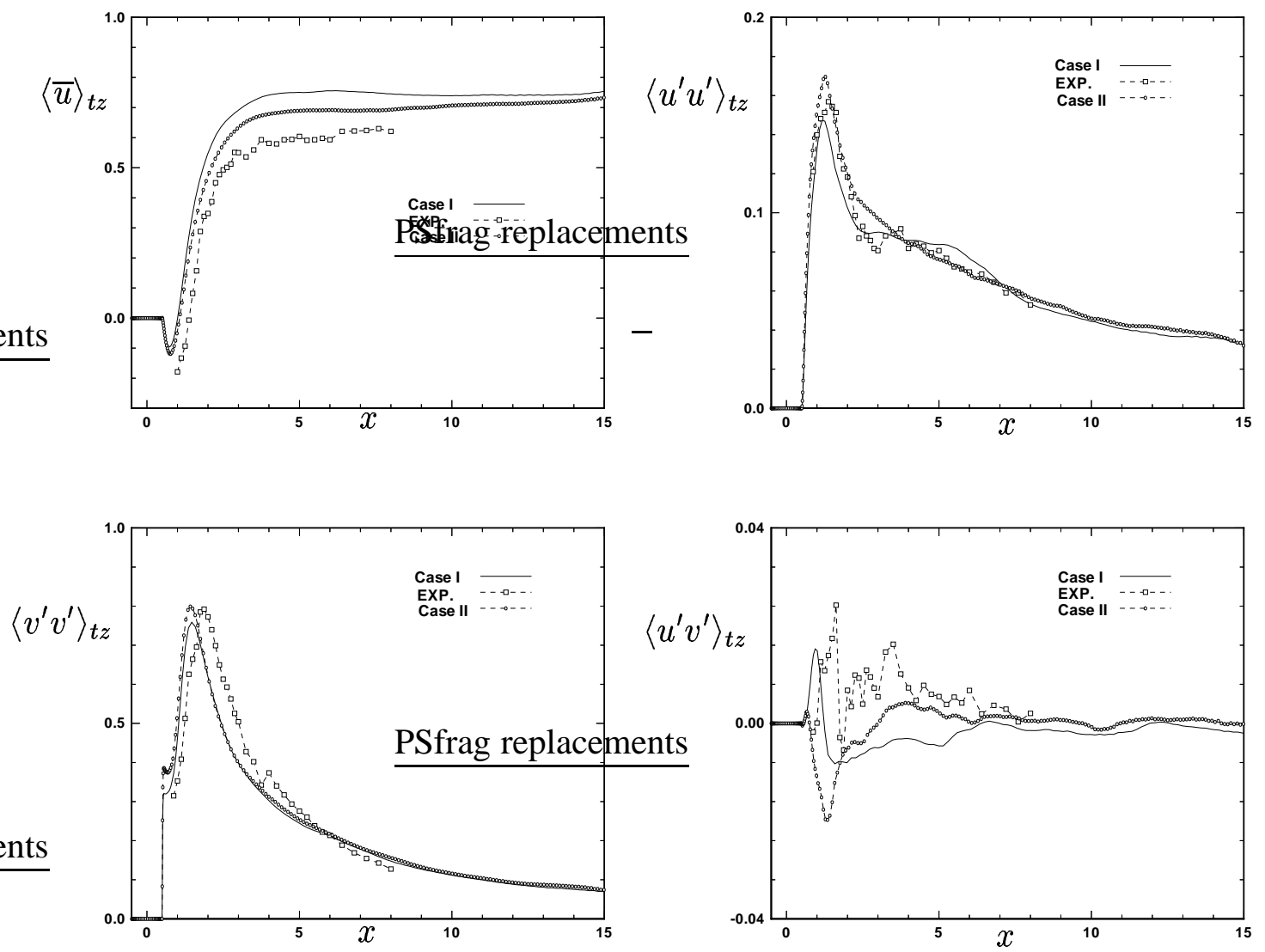

Figure 2: Time- and spanwise-averaged velocity, $\langle\bar{u}\rangle_{t z}$, and turbulent stresses $\left(\left\langle u^{\prime} u^{\prime}\right\rangle_{t z},\left\langle v^{\prime} v^{\prime}\right\rangle_{t z}\right.$, $\left.\left\langle u^{\prime} v^{\prime}\right\rangle_{t z}\right)$ at centerline $(y=0)$.
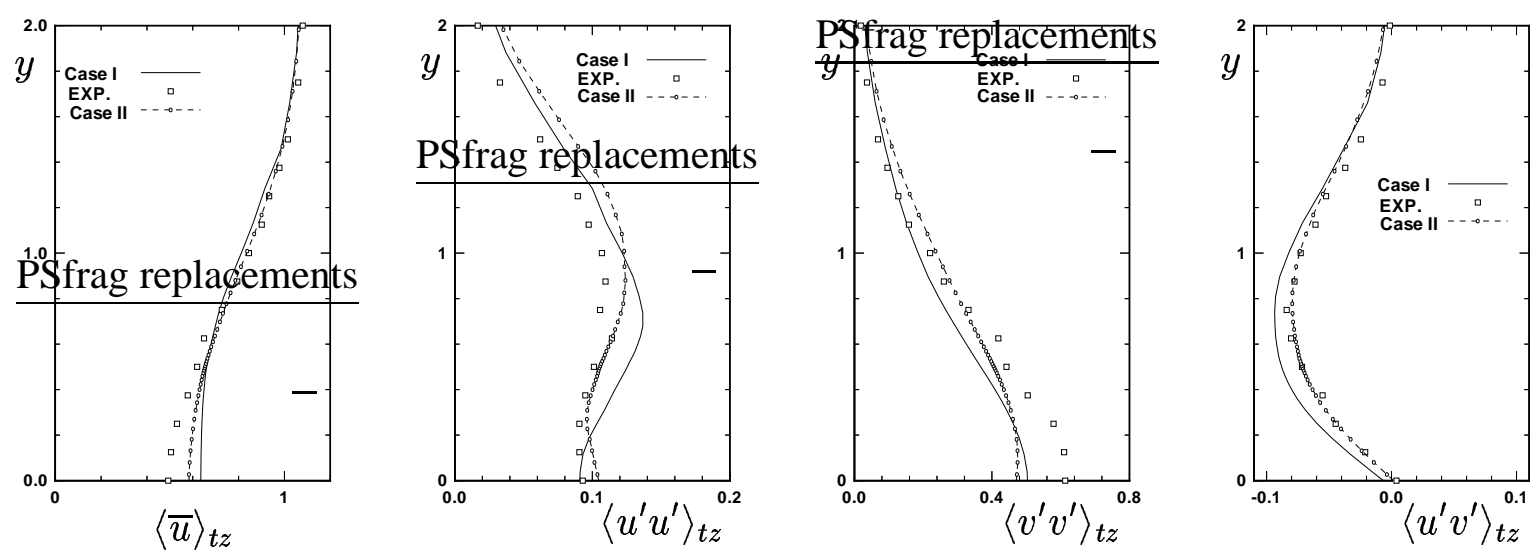

Figure 3: Time- and spanwise-averaged velocity, $\langle\bar{u}\rangle_{t z}$, and turbulent stresses $\left(\left\langle u^{\prime} u^{\prime}\right\rangle_{t z},\left\langle v^{\prime} v^{\prime}\right\rangle_{t z}\right.$, $\left.\left\langle u^{\prime} v^{\prime}\right\rangle_{t z}\right)$ versus $y$ at $x=2.5$. 

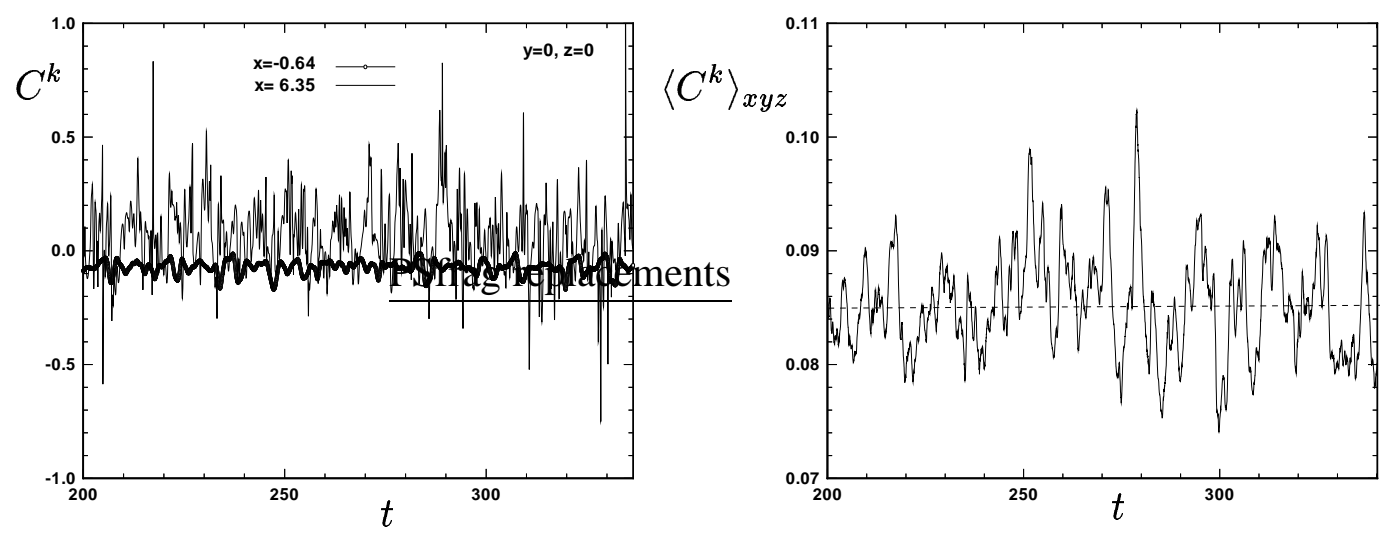

Figure 4: left) Time history of the $C^{k}$ at two chosen cells on the centerline, right) Time history of the $\left\langle C^{k}\right\rangle_{x y z}$.

[5] L. Davidson. In 2nd Int. Symp. on Turbulence Heat and Mass Transfer, 481-490, Delft, 1997.

[6] L. Davidson and P. Nielsen. In ROOMVENT'98, 1:125-132, Stockholm, 1998.

[7] M. Germano, U. Piomelli, P. Moin, and W.H. Cabot. Phys. Fluids A, 3:1760-1765, 1991.

[8] M. Germano, U. Piomelli, P. Moin, and W.H. Cabot. Erratum. Phys. Fluids A, 3:3128, 1991.

[9] S. Ghosal, T.S. Lund, P. Moin, and K. Akselvoll. Corrigendum. J. of Fluid Mech., 297:402, 1995.

[10] S. Ghosal, T.S. Lund, P. Moin, and K. Akselvoll. J. of Fluid Mech., 286:229-255, 1995.

[11] T. Igarashi. Bulletin of JSME, 27(231):1858-1865, September 1984.

[12] B. E. Lee. J. Fluid Mech., 69:263-282, 1975.

[13] D.A. Lyn, S. Einav, W. Rodi, and J.-H. Park. J. Fluid Mech., 304:285-319, 1995.

[14] D.A. Lyn and W. Rodi. J. Fluid Mech., 267:353-376, 1994.

[15] I. McLean and Gartshore. J. Wind Eng. Ind. Aero, 41:779-808, 1992.

[16] P. Moin and J. Kim. J. Fluid Mech., 118:341-377, 1982.

[17] C. Norberg. J. Wind Engng. and Ind. Aero., 49:187-196, 1993.

[18] A. Okajima. J. Fluid Mech., 123:379-398, 1982.

[19] U. Piomelli and T.A. Zang. Cr-187465 icase report no.90-80, NASA, 1990.

[20] W. Rodi. J. Wind Engng Ind. Aero., 69-71:55-75, 1997.

[21] W. Rodi. In New Tools in Turbulence Modelling. Springer Verlag, 1997.

[22] W. Rodi, J.H. Ferziger, M. Breuer, and M. Pourquié. J. Fluids Engineering, 119:248-262, 1997.

[23] J. Smagorinsky. Monthly Weather Review, 91:99-165, 1963.

[24] A. Sohankar. PhD thesis, Dept. of Thermo and Fluid Dynamics, Chalmers Univ. of Tech., 1998.

[25] A. Sohankar, L. Davidson, and C. Norberg. 12th Australasian Fluid Mech. Conf., 517-520, 1995.

[26] A. Sohankar, C. Norberg, and L. Davidson. J. Wind Engng Ind. Aero., 69-71:189-201, 1997.

[27] A. Sohankar, C. Norberg, and L. Davidson. Int. J. Num. Meth. Fluids, 26:39-56, 1998.

[28] A. Sohankar, C. Norberg, and L. Davidson. to appear in Phys. Fluids A, Feb. 1999.

[29] B. J. Vickery. J. Fluid Mech., 25:481-494, 1966.

[30] P. R. Voke. In J. P. Chollet et al., editor, Direct and Large Eddy Simulation II. Kluwer, 1997.

[31] C. H. K. Williamson. Annual Review of Fluid Mechanics, 28:477-539, 1996. 